\title{
An Improved Multihop Routing Protocol for Wireless Sensor Networks
}

\author{
Zhansheng Chen \\ School of Applied and Technology, Beijing Union University \\ School of Computer and Information Technology, Beijing Jiaotong University \\ Beijing, CHINA \\ ldtchenzs@buu.edu.cn
}

\begin{abstract}
Topology control in a sensor network balances load on sensor nodes and extends network lifetime. Clustering sensor nodes is an effective topology control approach. In this paper, an improved distributed clustering approach for wireless sensor network is proposed. Our proposed protocol, IMRP(Improved Multihop Routing Protocol), periodically selects cluster heads ac-cording to nodes intimacy, optimizes energy consumption among nodes in each clusters by using adaptive cluster head Round-Robin schema and balances energy consumption among cluster heads through a fitness routing algorithm based on a hybrid of the node residual energy and other secondary parameter, such as node proximity to its neighbors or radial angle. Simulation results show that the IMRP protocol effectively balances the energy consumption among nodes in a WSN network, eliminates the "hot spots" issues occurring in multihop routing protocols, and hence significantly increases network scalability and lifetime.
\end{abstract}

Keywords-wireless sensor networks; Routing protocols; Energy consumption; Clustering; Lifetime;

\section{INTRODUCTION}

Large scale wireless sensor networks (WSNs) rely on thousands of tiny sensor nodes to monitor targets, gather surrounding environmental information periodically and perform simple calculations. Nodes in a WSN communicate with each other within a given range in a self-organized manner, and send gathered environmental information to the base station for further processing through wireless transmission based on single-hop or multihop[1]. As a new complex system composed of information collection, processing and transmission, WSNs are widely used in military defense, environmental monitoring, biomedical, emergency rescue and disaster relief, as well as commercial applications [1].

In most cases, sensor nodes are deployed randomly in many inaccessible places by throwing from airplanes, and its energy is provided by batteries with a life expectation of months or even years under harsh conditions. Accordingly, how to design a protocol which can effectively reduces node's energy consumption and prolong the network's lifetime becomes a hot research topic [2].

\section{RELATED WORK}

A number of protocols based on hierarchical techniques have been proposed by many research scholars[3], [4], which can aid in reducing energy consumption. The essential operation of hierarchical techniques in sensor node clustering is to select a set of cluster heads from the set of nodes in the WSN network, and then cluster the remaining nodes with these heads.

Typical hierarchical routing protocols include LEACH (low energy adaptive clustering hierarchy)[5], and HEED(hybrid, energy-efficient, distributed)[6], etc. LEACH is the first cluster-based routing protocol proposed by Heinzelman in WSNs. Its basic idea is to elect a cluster head periodically by equal probability round-robin method, realize sensor node energy consumption balance as close as possible throughout the WSN, reduce nodes energy consumption effectively and prolong the lifecycle of WSN network. HEED[6] clustering algorithms proposed by Younis etc. generate more uniform clusters through a number of iterations, balance the nodes energy consumption and prolong the network lifetime, but its cluster build process is too complicate. Energy-Aware Routing algorithm [7] presented by Xiao-Hui $\mathrm{Li}$ etc. uses local betweenness centrality to estimate the energy consumption of the neighboring nodes around a given local sensor node, without global information about the network topology. Q-LEACH [8] protocol is put forward by B. Manzoor etc. for homogenous networks, significantly improved network parameters and seems to be an attractive choice for WSNs. BEENISH [9] proposed by T. N. Qureshi etc. assumes WSN containing four energy levels of nodes, cluster heads are elected on the bases of residual energy level of nodes. An energy-efficient prediction clustering algorithms is put forward by Jian Peng etc[10], enables the nodes to select the cluster head according to factors such as energy and communication cost, so that the network energy can be dissipated uniformly. Energy-Efficient node selection scheme based on genetic algorithms is proposed[11], which can optimize the tradeoff between the accuracy of tracking and the energy cost of nodes.

The remainder of this paper is organized as follows: Section 3 describes the network model and states the problem that we address in this work. Section 4 presents the IMRP protocol and argues that it satisfies its objectives. Section 5 shows IMRP effectiveness via simulations and compares it to LEACH and DIRECT protocols. Section 6 gives concluding remarks and directions for future work. 


\section{Problem Statement}

Cluster-based routing protocol for wireless sensor network is closely related to specific application scenarios. Monitoring tasks depend on the type of monitoring environmental, but their's essence are all the same, deployed a number of sensor nodes randomly within a given region by throwing manner. The following section describes the network model, transimission model, data fusion model and run-time model.

\section{A. Network Model}

Consider a set of sensors dispersed in a field as [12] which length is $\mathrm{X}$ and width is $\mathrm{Y}$, area is $\mathrm{X} \times \mathrm{Y}$, sink's coordinates is $(X / 2,3 Y / 2)$, distribution of network nodes as shown in Figure 1.Assume the sensor nodes are left unattended after deployment. The sensor nodes are quasi-stationary, have the same limited initial energy, similar capabilities including processing and communication and equal significance. All sensor nodes have a unique ID, know their own coordinate information. Links are symmetric, two nodes can communicate with each other using the same transmission power level. Each node has a fixed number of controlling transmission power level. All sensor nodes have the ability to compute the distance between two nodes based on received signal strength. All sensor nodes have data fusion ability and transmit data periodically.

\section{B. Transmission Model}

IMRP protocol uses the same wireless communication model which is similar to that of [5] , in which the energy transmit and receive formulas are equation 1 and equation 2 :

$$
\begin{gathered}
E_{T x}(k, d)=\left\{\begin{array}{l}
k E_{\text {elec }}+k \varepsilon_{\text {fs }} d^{2}, d<d_{0} \\
k E_{\text {elec }}+k \varepsilon_{m p} d^{4}, d>d_{0}
\end{array}\right. \\
\mathrm{E}_{R x}(k, d)=E_{\text {elec }}(k)
\end{gathered}
$$

\section{Data Fusion Model}

IMRP protocol reduces network data traffic through data fusion technology, so as to achieve the goal of saving energy consumption performance. We assume that all nodes have the same perception capability, cluster head receive $\mathrm{k}$ bits data information from other nodes, compress into $\mathrm{k}$ bits data, and the energy consumption of data fusion $E_{D A}$ is $5 \mathrm{~nJ}=$ bit.

\section{IMRP RunTime Model}

IMRP protocol uses "round" as network runtime unit based on LEACH Protocol, which is divided into cluster build stage and cluster running stage. Network runtime distribution model as shown in Figure 1.

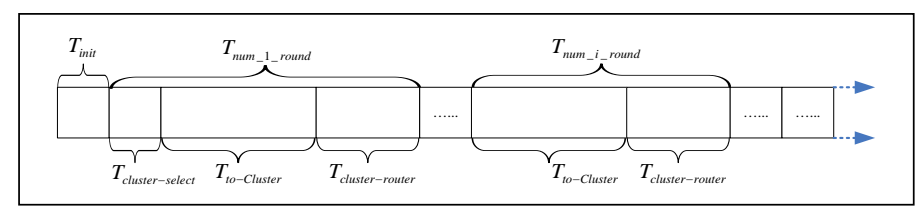

Fig. 1. Running-Time model of IMRP
Themselves into local clusters. According to the ratio of total rest energy to total initial energy in WSN network, we determine the specific value $\mathrm{m}$ of cluster round dynamically, so as to achieve the goal of saving energy consumption.

\section{IMRP PROTOCOL : IMPROVED MULTIHOP ROUTING PROTOCOL}

\section{A. IMRP Network Initialization}

Let the distance intimacy between nodes be the measure principle of clustering in the IMRP network initialize stage in this article. The distance computing formula between node $\mathrm{S}_{\mathrm{i}}\left(\mathrm{x}_{\mathrm{i}}, \mathrm{y}_{\mathrm{i}}\right)$ and node $\mathrm{S}_{\mathrm{j}}\left(\mathrm{x}_{\mathrm{j}}, \mathrm{y}_{\mathrm{j}}\right)$ as shown in formula 3 .

$$
d_{i j}=-\sqrt{\left(x_{i}-x_{j}\right)^{2}+\left(y_{i}-y_{j}\right)^{2}}, i \neq j
$$

In formula 3 , let the distance between node $i$ and node $j$ be negative, which means the bigger the value is, the higher the intimacy is. IMRP network starts into cluster head election phase when sink issues synchronous command.

\section{B. IMRP M-round Cluster phase}

In order to balance nodes energy consumption, residual energy method was used in the [13] for improving LEACH protocols, which have achieved certain better results. WSN monitoring region first be divided into clusters using the AP propagation algorithm in IMRP protocol, followed by cluster head election and send data to sink by cluster head through multihop routing manner.

1) IMRP M-round Cluster Head Election Method: At the cluster head election phase, cluster head election threshold refers to the node residual energy, the distance between node and Sink node, and the frequency of node not act as a cluster head in IMRP protocol.The introduction of these factors is expected to improve overall energy consumption balance in network, as shown in formula 4 and formula 5.

$$
\begin{gathered}
T(s)_{\text {IMRP }}=\frac{p}{1-p(r \bmod (1 / p)} \times f \\
f=\frac{E_{r_{-} \text {cur }}}{E_{0}}+\frac{d_{\text {to_Sink }}}{\sum_{k=1}^{C_{i-\text { num }}} d_{k_{-} t o \_} \text {Sink }}+r_{m} \operatorname{div} \frac{1}{p}
\end{gathered}
$$

Let $\mathrm{E}_{\mathrm{r}_{\text {ccur }}}$ denote the residual energy of node in the current round, $\mathrm{C}_{\mathrm{i} \_ \text {num }}$ is the number of nodes within the cluster range, $\mathrm{d}_{\mathrm{to} \text { Sink }}$ denote the distance between node and Sink, $\mathrm{r}_{\mathrm{m}}$ denote the number of round that node does not act as cluster head continuously.

2) Cluster Announce Information: After all cluster heads was elected, cluster heads need to declare the control messages of became cluster head in the cluster. The control message mainly contains the location attribute etc. Since the coverage of each cluster is different, the maximum transmit power depends on the maximum distance between the cluster head and all cluster member in the cluster, and the calculation formula of maximum distance as shown in formula 6 .

$$
d_{C_{-} i_{-} \max }=\max \left\{d_{C_{-} i k}\right\} \quad k=1,2,3, \ldots, C_{i_{\text {num }}}
$$


3) IMRP m-round Self-organizing network topology: The basic idea is it determines the cluster head first according to the election algorithm, and runs the data collection and routing delivery continuously in the m-1 rounds, which remains the cluster head unchanged.

\section{IMRP M-round Cluster phase}

1) IMRP Inner-cluster Communication: Cluster heads are responsible for coordination among the nodes within their clusters and aggregation of their data. The status of node including active state and dormant state, which can be known from literature [14]. This paper will use the scheme that combines node dormancy mechanism and TDMA(Time Division Multiple Access)technology to address WSN data collection problem periodically.

2) IMRP Inter-cluster Communication: Cluster heads are also responsible for communication with each other. In this paper, we use CDMA technology to assign a unique code to each cluster to avoid signal interference problem. The energy consumption of cluster heads are not balance due to the difference of the distance between the cluster head and the sink. In order to address this problem, we use the strategy combining the single-hop routing and the multihop routing based on the LEACH protocol. Specific routing policy is as follows: the entire network is layered logically in the first place, the specific layer formula as shown in formula 7 and formula 8 .

$$
\begin{gathered}
\mathrm{CH}_{i_{-} \text {layer }}=\left(d_{\mathrm{CH}_{i_{-}} \operatorname{Sink}} \operatorname{div} d_{0}\right)+1 \\
d_{0}=\sqrt{e f s / e m p} \quad i=1,2,3, \ldots, N_{C H}
\end{gathered}
$$

In formula 7, $\mathrm{NCH}$ denotes the number of cluster heads. Here, after the network logical layering, we are going to consider two kinds of data communication model as follows.

\section{a) Solo-layer cluster head data communication model}

The cluster head that located at layer one should communication with the Sink directly by using single-hop pattern.

b) Multi-layer cluster head data communication model

The cluster head that located at two or higher logic layer should communication with the Sink indirectly by using multihop pattern. The routing strategies of how cluster head is selected as the next hop is described as shown in formula (9).

$$
f=\lambda_{1}\left(\frac{E_{c h_{-} \text {cur_res }}}{E_{0}}\right)+\lambda_{2}\left(\frac{d_{0}-d_{i, j}}{d_{0}}\right)+\lambda_{3}\left(\frac{1}{\cos \theta}\right)+\lambda_{4}\left(\frac{E_{c h \_c u r}}{\sum_{k=1}^{c h_{\text {num }}} E_{c h_{k_{-}} \text {cur }}}\right)
$$

In formula 9, $\mathrm{E}_{\mathrm{ch} \_ \text {cur_res }}$ denotes the residual energy of cluster head; $\lambda_{i}(i=1,2,3,4)$ denotes the weight coefficient of each factor; $\theta$ shows the deflection angle of data transfer.

3) Communication between Sink and network nodes: The main task of sink is to coordinate the cluster build and data communication among nodes through control message in network.

\section{Protocol Simulation And Result Analysis}

\section{A. Simulation Environment}

This paper uses MATLAB as a simulation platform, monit- oring scenario for $100 \mathrm{~m} \times 100 \mathrm{~m} 2 \mathrm{~d}$ plane, 100 nodes deployed randomly in monitoring scope, specific parameters in the simulation are shown in Table I.

TABLE I. EXPERIMENTAL PARAMETER IN SimUlated PROTOCOL

\begin{tabular}{|c|c|c|c|}
\hline Parameter & value & Parameter & value \\
\hline$E_{\text {elec }}$ & $5 \mathrm{~nJ} / \mathrm{bit}$ & Data_size & $2000 \mathrm{bit}$ \\
\hline$e f s$ & $10 \mathrm{~nJ} / \mathrm{bit} / \mathrm{m}^{2}$ & Control_size & $100 \mathrm{bit}$ \\
\hline$p$ & 0.05 & $n$ & $100(200 / 400 / 600)$ \\
\hline$e m p$ & $0.0013 \mathrm{~nJ} / \mathrm{bit} / \mathrm{m}^{2}$ & $E_{0}($ unit:J) & $0.5(0.01 / 0.02 / 0.05 / 1.0)$ \\
\hline$d_{0}$ & sqrt(efs/emp) & $X$-Max(m) & $100(200 / 400 / 800)$ \\
\hline$E_{D A}$ & $5 \mathrm{~nJ} / \mathrm{bit} / \mathrm{message}$ & $Y$-Max(m) & $100(200 / 400 / 800)$ \\
\hline
\end{tabular}

\section{B. Performance comparison}

1) Cluster formation result

In this paper, AP(Affinity Propagation) algorithm is used to divide the network into clusters, and cluster formation final result is shown in Figure 2.

2) Round number of survival nodes

Figure 3 depicts network lifetime with IMRP protocol which is longer than that of LEACH and DIRECT in the same percent of survival nodes.

It is assumed that network lifetime can be defined as the round number elapsed until the first node depletes its energy. In terms of the first node failure, IMRP protocol's 1896 rounds if far longer than 1449 rounds in LEACH and 328 rounds in DIRECT. At the percent of $80 \%$, three protocols are 2353 , 1855 and 2192 rounds respectively. After 100 times of random simulation, experiment result shows that the network lifetime of IMRP rises up to $30.9 \%$ extension in network lifetime compared with LEACH and rises up to $478 \%$ extension in network lifetime compared with DIRECT.

3) Average energy consumption in clusters

Simulation results show that average energy consumption rate of clusters are very close, so IMRP protocol effectively balance the energy consumption between different clusters.

From figure 4 we find the cluster which is located in the center of the network failure earlier, the reason is that the cluster burden the routing forward role earlier and frequently.

\section{4) Average energy consumption in clusters}

Simulation results show that average energy consumption rate of clusters are very close, so IMRP protocol effectively balance the energy consumption between different clusters.

From figure 4 we find the cluster which is located in the center of the network failure earlier, the reason is that the cluster burden the routing forward role earlier and frequently.

\section{5) Time span of nodes failure}

Figure 5 shows the round number of three protocols in different percent nodes failure. Simulation results show that under the condition of the same number of failure nodes, the round number of IMRP protocol is far behind LEACH and DIRECT protocol, suggesting that IMRP protocol improves the balance of energy dissipation among nodes. 

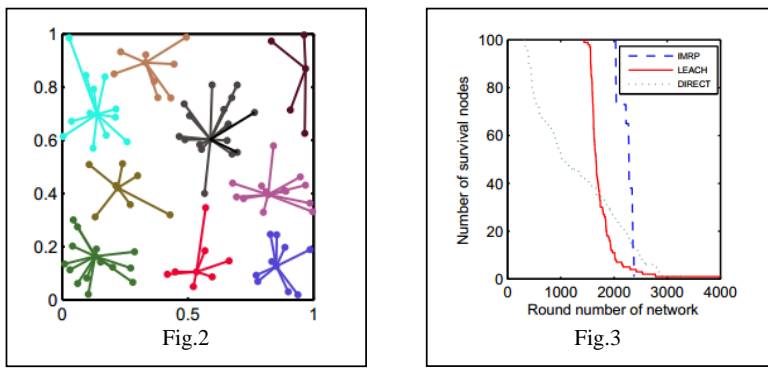

Fig. 2. Cluster formation results of AP

Fig. 3. Round number of survival nodes in three protocols
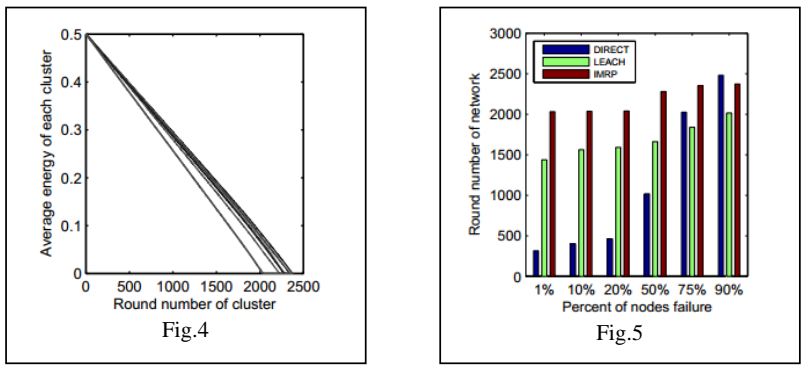

Fig. 4. Energy consume ratio of clusters in IMRP

Fig. 5. The round number in different percent nodes failure

\section{6) Correlation Analysis of Node Initial Energy}

Under the condition of keeping other parameters constant, we change the node initial energy $\mathrm{E}_{0}$ of network and simulation using MATLAB. Figure 6 shows the simulation results of the network lifetime when nodes initial energy are $0.02 \mathrm{~J}, 0.05 \mathrm{~J}, 0.1 \mathrm{~J}$ and $1.0 \mathrm{~J}$ respectively.

Simulation results show that IMRP protocol has universality to the different initial energy of nodes on the same simulation conditions, and has better energy saving features, better scalability, better convergence rate and better stability than LEACH and DIRECT.

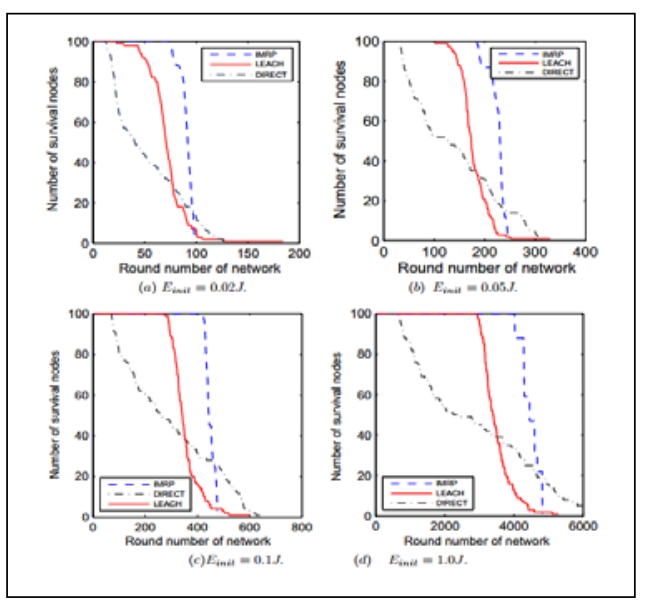

Fig. 6. Round number of survival nodes in three protocols when Node initial energy is not the same.

\section{CONCLUSION}

Due to the uneven energy consumption problem of nodes in a wireless sensor network, an improved energy efficient multihop routing protocol based on clustering (IMRP) is proposed in this paper. In comparison with LEACH and DIRECT protocols, IMRP can produce relatively even distributed clusters and reduce the traffic load both intracluster and inter-clusters. Simulation results show that the IMRP protocol effectively balances the energy consumption of the whole WSN network, prolongs the network lifetime, and has good scalability and better convergence rate.

\section{References}

[1] Zhang Xiao-ling, LIANG Wei, YU Hai-bin, et al. Survey of transmission scheduling methods in wireless sensor networks. Journal on Communications, 2012, 33(5):143-157.

[2] Ma ZC, Sun YN, Mei T. Survey on wireless sensors network. Journal of China Institute of Communications, 2004, 25(4):114-124(in Chinese with English abstract).

[3] Nikolaos A, Pantazis, Stefanos A, et al. Energy-Efficient Routing Protocols in Wireless Sensor Networks: A Survey. In: IEEE COMMUNICATIONS SURVEYS \& TUTORIALS, 2013, 15(2):551591

[4] LI B, WANG W J, YIN Q Y, et al . An energy-efficient geographic routing based on cooperative transmission in wireless sensor networks[J] Sci China Inf Sci, 2013, 56: 4757-4762.

[5] Heinzelman WR, Chandrakasan A, Balakrishnan H. Energy-Efficient communication protocol for wireless microsensor networks. In: Proc. of the Hawaii Int'l Conf. on System Sciences. San Francisco: IEEE Computer Society, 2000. 3005-3014.

[6] Younis O, Fahmy S. Distributed clustering in ad-hoc sensor networks: A hybrid, energy-efficient approach. In: Zhang ZS, Low S, eds. Proc. of the IEEE INFOCOM. San Francisco: IEEE Computer Society Press, 2004.

[7] Xiao-Hui Li, Zhi-Hong Guan. Energy-Aware Routing in Wireless Sensor Networks Using Local Betweenness Centrality[J]. International Journal of Distributed Sensor Networks, 2013:1-9.

[8] B. Manzoor, N. Javaid, O. Rehman, et al. Q-LEACH: A New Routing Protocol for WSNs, Procedia Computer Science, 2013, 926-931.

[9] T. N. Qureshi, N. Javaid, A. H. Khan, et al. BEENISH: Balanced Energy Efficient Network Integrated Super Heterogeneous Protocol for Wireless Sensor Networks, Procedia Computer Science, 2013:920-925.

[10] Jian Peng, Tang Liu, Hongyou Li, et al. Energy-Efficient Prediciton Clustering Algorithm for Multilevel heterogeneous Wireless Sensor Networks[J]. International Journal of Distributed Sensor Networks, 2013:1-8.

[11] Yong Wang and Dianhong Wang. Energy-Efficient Node Selection for Target Tracking in Wireless Sensor Networks[J]. International Journal of Distributed Sensor Networks, 2013:1-6.

[12] JIANG C J, SHI W R, TANG X L, et al . Energy-balanced unequal clustering routing protocol for wireless sensor networks[J]. Journal of Software, 2012, 23(5):190-200.

[13] CHEN QZ, Zhao XM, Chen XY. Design of double rounds clustering protocol for improving energy efficient in wireless sensor networks. Journal of Software, 2010, 21(11):2933-2943.

[14] Ngo HQ, Lee YK, Lee SY. MEPA: A new protocol for energy-efficient, distributed clustering in wireless sensor networks. In: Proc. of the IEEE Int'l Symp. on Wireless Communication Systems. Norway: Wireless Communication Systems, 2007. 40-44. 